\title{
Determinant role for the gep oncogenes, Ga12/13, in ovarian cancer cell proliferation and xenograft tumor growth
}

\author{
Ji Hee Ha ${ }^{1, *}$, Rohini Gomathinayagam ${ }^{1, *}$, Mingda Yan ${ }^{1}$, Muralidharan Jayaraman ${ }^{1}$, \\ Rajagopal Ramesh², and Danny N. Dhanasekaran ${ }^{1}$ \\ ${ }^{1}$ Stephenson Cancer Center and the Department of Cell Biology, The University of Oklahoma Health Sciences Center, \\ Oklahoma City, OK, USA \\ ${ }^{2}$ Stephenson Cancer Center and the Department of Pathology, The University of Oklahoma Health Sciences Center, Oklahoma \\ City, OK, USA \\ * These authors contributed equally
}

Correspondence to: Danny N. Dhanasekaran, email: danny-dhanasekaran@ouhsc.edu Keywords: G proteins, ovarian cancer, Ga12, Ga13, gep, gip2, oncogenes

Received: July 09, 2015

Accepted: July 30, 2015

Published: August 03, 2015

This is an open-access article distributed under the terms of the Creative Commons Attribution License, which permits unrestricted use, distribution, and reproduction in any medium, provided the original author and source are credited.

\section{ABSTRACT}

Recent studies have shown that the gip2 and gep oncogenes defined by the a-subunits of Gi2 and G12 family of G proteins, namely Gai2 and Ga12/13, stimulate oncogenic signaling pathways in cancer cells including those derived from ovarian cancer. However, the critical a-subunit involved in ovarian cancer growth and progression in vivo remains to be identified. Using SKOV3 cells in which the expressions of individual Ga-subunits were silenced, we demonstrate that the silencing of Ga12 and Ga13 drastically attenuated serum- or lysophosphatidic acid-stimulated proliferation. In contrast, the invasive migration of these cells were reduced only by the silencing of Gai2 or Ga13. Analyses of the xenograft tumors derived from these Ga-silenced cells indicated that only the silencing of Ga13 drastically reduced xenograft tumor growth and prolonged the survival of the mice. Similar, but albeit reduced, effect was seen with the silencing of Ga12. On the contrary, the silencing of Gai2 or Gaq failed to exert such effect. Thus, our studies establish for the first time that Ga12/13, the putative gep oncogenes, are the determinant a-subunits involved in ovarian cancer growth in vivo and their increased oncogenicity can be correlated with its ability to stimulate both proliferation and invasive migration.

\section{INTRODUCTION}

Detection of ovarian cancer at the early stages is still a challenge, and only about $15 \%$ of the patients get to be diagnosed at the earliest. Alarmingly, about $61 \%$ of the ovarian cancer patients are diagnosed at the therapeutically challenging, metastasized stages of cancer, leading to a poor prognosis and survival. The $2015 \mathrm{NCI}$, SEER statistics indicates that approximately 21,290 women will newly be diagnosed with ovarian cancer and about 14,180 ovarian cancer patients will die of the disease [1] Such statistical evidence underscore the need for the identification of better diagnostic, prognostic and therapeutic targets for the management of ovarian cancer.

Tumor genesis and progression are mediated by aberrant, and asynchronous signaling networks involving multitudes of receptors and their downstream signaling nodes. Oncogenic signaling nodes involving receptor tyrosine kinases and cytokines are well characterized to a large extent. However, only quite recently, the oncogenic potential of $\mathrm{G} \alpha$-subunits that primarily transmit signaling from their cognate $G$ protein coupled receptors is beginning to be realized. Tumor promoting activities of GPCRs such as those of thrombin [2], Sphingosine-1-phosphate [3, 4], Prostaglandins [5], and lysophosphatidic acid (LPA) have ben shown to be associated with the activation of specific $\alpha$-subunits [6] [7][8, 9]. These $\alpha$-subunits, especially those of Gai- and Ga12-family, have also been identified to transduce signaling from chemokine family of GPCRs to diverse oncogenic responses underlying tumor promotion and metastasis [10-15] [16, 17]. Due to intrinsic potential of 
activating tumorigenic pathways, the activated forms of Gai2 and Ga12/13 subunits are referred to as gip2 and gep oncogenes [18]. In addition to transmitting signals from GPCRs, it has also been identified that these $\alpha$-subunits act as critical signaling hubs to transduce growth promoting activities from receptor tyrosine kinases [19-22], wnt signaling [22, 23], sonic hedgehog signaling [24-28], hippo signaling [28, 29], and steroid hormone receptors $[30,31]$. While these studies point to the potential role of $\mathrm{G} \alpha$-subunits in cancer genesis and progression, the identity of specific $\alpha$-subunit(s) involved in promoting tumor growth in a defined cancer context remains to be established.

In such a scenario, it is significant to note that ovarian cancer patients show elevated levels of LPA and the resultant aberrant signaling by LPA-receptors (LPARs) has been correlated with increased cell proliferation, migration, and neovasculogenesis in cancer [32-34]. While these in vitro observations clearly implicate specific G $\alpha$-subunit(s), downstream of LPA-LPAR signaling, the identity of the G $\alpha$-subunit that could promote tumor growth in vivo has not been defined. Although Gai-, Gaq-, and Ga12-family of proteins have been shown to transduce mitogenic as well as motogenic signals from LPARs in ovarian cancer cells $[4,35,36]$, a comparative analysis to identify the $\mathrm{G} \alpha$-subunit involved in stimulating ovarian cancer growth in vivo has not been undertaken until now. Therefore, in the present study, we examined

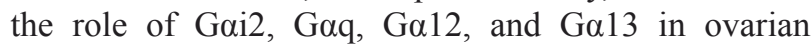
cancer cell proliferation and migration in vitro and their tumorigenic role in xenograft tumors in vivo, by utilizing SKOV3 cells expressing nonspecific scrambled shRNA (SKOV3 NS) control cells and the respective $\mathrm{G} \alpha$-silenced SKOV3 cells (shGai2, shGaq shGa12, and shGa13). Our results demonstrate that the silencing of $\mathrm{G} \alpha 12$ and $\mathrm{G} \alpha 13$ attenuates LPA-mediated proliferation of SKOV3 cells, thus establishing a mitogenic role for these $\alpha$-subunits. We demonstrate further that the migratory potential and the invasive migration of these cells are reduced upon the

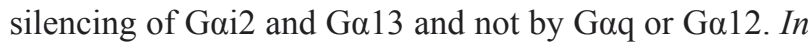
vivo analyses of xenograft tumor growth results indicate that the silencing of G $\alpha 13$ drastically reduced xenograft tumor growth and prolonged survival of the mice. While the silencing of G $\alpha 12$ exerts a similar, but rather slightly reduced effect, the silencing of Gai2 or Gaq failed to show any protective advantage for the tumor bearing mice. Thus, our studies establish for the first time that Ga13 is the primary $\alpha$-subunit involved in accelerating ovarian cancer growth, in vivo, and the increased oncogenicity of $\mathrm{G} \alpha 13$ can be correlated with its ability to costimulate the signaling nodes involved in proliferation and invasive migration.

\section{RESULTS AND DISCUSSION}

Oncogenic phenotypes in cancer cells are manifested by an increase in the rate of cell proliferation along with a heightened migratory and invasive potential. GPCRs such as LPARs, PARs, CXCRs, and CCRs transmit their oncogenic signaling in cancer cells primarily through Gai2, Gaq, G $\alpha 12 / \mathrm{G} \alpha 13$ in a context specific manner. Additionally, in many instances, these G $\alpha$-subunits have also been shown to transmit signaling from non-GPCRs family of receptors in a context specific manner [21, 38, 39]. Therefore, defining the oncogenic potential of these $\alpha$-subunits has become crucial for the development of highly efficient therapeutics for cancer. This is of greater significance in the case of ovarian cancer in which LPA, which can activate all of these Go-subunits, plays a major role in the mitogenic and motogenic pathways underlying the disease progression. With this reasoning, we sought to evaluate the cell proliferative, migratory, invasive, and/or tumor-promoting potential of each of these $\alpha$-subunits. First, we investigated the relative ability of these $\alpha$-subunits to stimulate cell proliferation in an in vitro assay. Proliferation of SKOV3 cells in which the expression of a specific $\mathrm{G} \alpha$-subunit had been silenced was first monitored by an automated cell enumeration assay using Operetta High Content System. Results from this assay indicated that LPA as well as FBS stimulated an increase in cell number by $48 \mathrm{hrs}$ and this

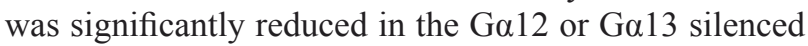
cells (Figure 1A). We also monitored the proliferation of these cells using a S-phase cell labeling method that measures the incorporation 5-ethynyl-2'-deoxyuridine into DNA. As shown in Figure 1 (A, B, C), results from both the cell count based and the $S$ phase labeling analysis indicated that the proliferation of these cells were

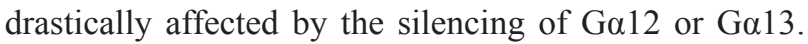
More significantly, silencing of Gai2 and Gaq failed to have any such inhibitory effect on LPA or serum mediated proliferation of these cells. While the results obtained

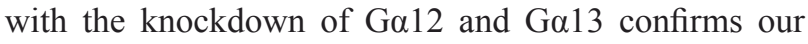
previous findings that LPA as well as serum-stimulated proliferation of ovarian cancer cells is primarily mediated by $\mathrm{G} \alpha 12$ and $\mathrm{G} \alpha 13$ [33, 40, 41], the corollary findings that cell proliferation is not affected by the silencing of Gai2 or Gaq, firmly establishes the unique role of these gep oncogenes in LPA and serum mediated proliferation of ovarian cancer cells.

Next, we evaluated the ability of these $\alpha$-subunits to confer migratory potential to SKOV3 cells, by testing whether the silencing of any of the $\alpha$-subunits attenuate serum or LPA-mediated migration of these cells. A migration assay using live cell imaging in Operetta High Content Screening system was used to monitor the effect 


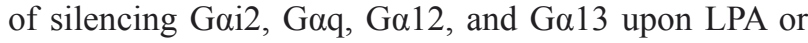
serum stimulated migration of SKOV3 cells. Our results indicated that the silencing of Gai2 or Ga13 drastically reduced LPA/serum-stimulated migration by 68 and 39 $\%$ respectively. Silencing of G $\alpha 12$ and Gaq did not have any such attenuating effect. Thus, contrary to the results obtained with proliferation studies, these results point to a dominant role for Gai2 and Ga13 in LPA as well as FBS induced cancer cell migration. In addition to an intrinsic increase in migratory potential, cancer cells also exhibit an invasive phenotype. Therefore, we investigated whether Gai2 and Ga13 have similar effects on the invasive migration of SKOV3 cells. Respective G $\alpha$-silenced SKOV3 cells along with scrambled shRNA expressing control cells were evaluated for their ability to inhibit LPA or FBS stimulated invasive migration of ovarian cancer cells, using a collagen-coated Transwell based invasive cell migration assay. Our results indicated that the invasive potential was attenuated significantly again

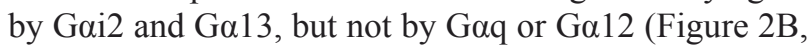
C). The silencing of Gai2 attenuated LPA-stimulated cell migration by $97.6 \%$ and FBS-stimulated cell migration by $74.1 \%$. In a similar fashion, the silencing of $\mathrm{Ga} 13$ reduced LPA-stimulated invasive migration it $73.8 \%$ and FBS-stimulated invasive migration by $89.1 \%$. Although the silencing of $\mathrm{G} \alpha 12$ or Gaq failed to have any effect

Figure 1: Effect of silencing $G \alpha$-subunits in the proliferation of SKOV3 cells. (A) SKOV3 in which the individual $\mathrm{G} \alpha$-subunits were silenced (shGai2, shGoq, shGo12, or shGa13) were plated in 96-well plates $\left(5 \times 10^{3}\right.$ cells/well) along with the cells expressing scrambled shRNA (shNS). Cell were serum starved for 18 hours, and stimulated with $10 \%$ FBS or $20 \mu \mathrm{M}$ LPA. Cell numbers were determined at 48 hours by live cell imaging in an Operetta HCS imaging analyzer by digital phase contrast imaging and cell count analysis using the Harmony image analysis software. Results are presented as the percent change over control values using the values derived from cells expressing scrambled shRNA as the control. Experiment was repeated thrice and the results are presented as mean $\pm \mathrm{SEM}$. Significance was calculated by Student's-t test $\left({ }^{*} p<0.05,{ }^{* *} p<0.01\right)$. (B) SKOV cells expressing scrambled shRNA (shNS) and cells in which the individual $\mathrm{G} \alpha$-subunit were silenced were plated in a 96 -well plate $\left(5 \times 10^{3}\right.$ cells/well). Serum starved cells were stimulated with $10 \% \mathrm{FBS}$, or $20 \mu \mathrm{M}$ LPA for $48 \mathrm{hrs}$. Proliferating S-phase cells were imaged using Click-iT Plus EdU Alexa Fluor 488 imaging kit that monitors 5-ethynyl2'-deoxyuridine incorporation into DNA. Proliferating cells (green) versus the total number of DAPI-labeled cells (blue) were imaged imaged using Operetta HCS system. (C) Proliferating cells versus the total number of DAPIlabeled cells were quantified in Operetta System using the Harmony image analysis software. Results are presented as percent change over the shNS control values (Mean \pm SEM; $\mathrm{n}=3 ; * \mathrm{p}<0.05, * * \mathrm{p}<0.01)$. on FBS-stimulated invasive migration, the silencing of Ga12 reduced LPA-stimulated migration by $19 \%$. Rather surprisingly, the silencing of Gaq appears to promote overall invasive migration as shown by the serum starved as well as serum-stimulated shGaq cells (Figure $2 \mathrm{C}$, D). Although the underlying mechanism is not known at present, at least these results rule out a role for Gaq in

A

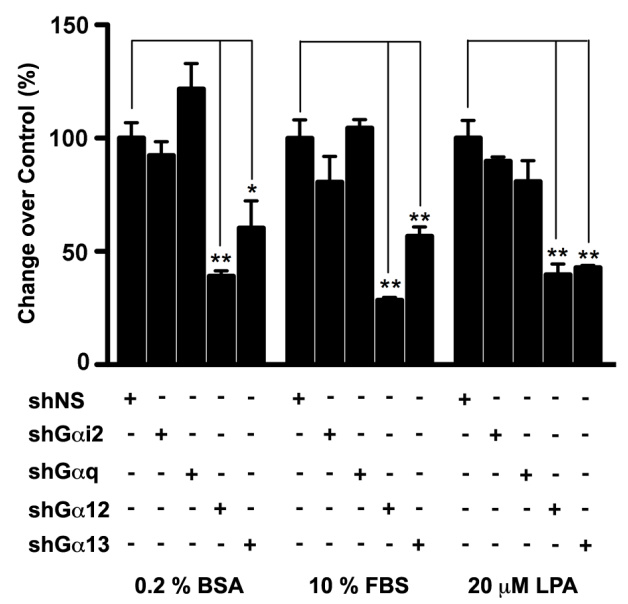

B

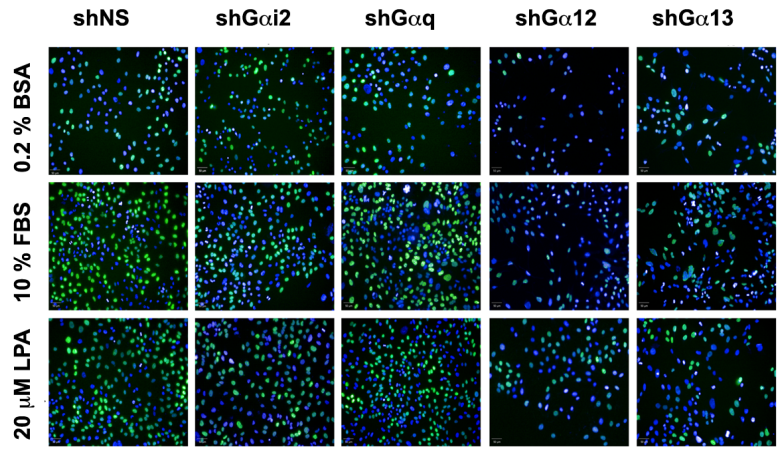

C

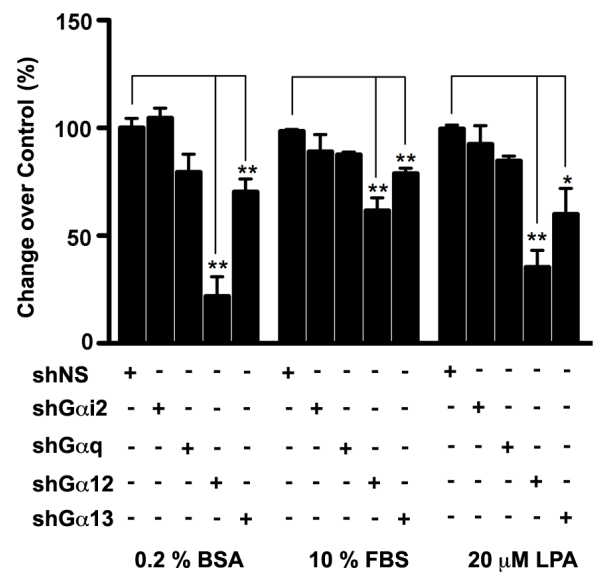


the invasive migration of ovarian cancer cells. Thus far, there have been contradicting reports on the identity of the $\mathrm{G} \alpha$-protein involved in LPA- or serum-mediated invasive migration of ovarian cancer cells. In this context, our comparative analysis to identify the role of a specific $\mathrm{G} \alpha$ protein has finally established that both Gai2 and Ga13 are involved in promoting cell migration in ovarian cancer cells. It is likely that the spatiotemporal coordination of signaling inputs from both of these $\alpha$-subunits is involved in the invasive migration of ovarian cancer cells. Further, our observation that Gai2-Rac as well as Ga13-Rhomediated pathways are required for LPA mediated invasive migration of ovarian cancer cells $[36,42]$ as well as the independent findings that the migration mediated by
A
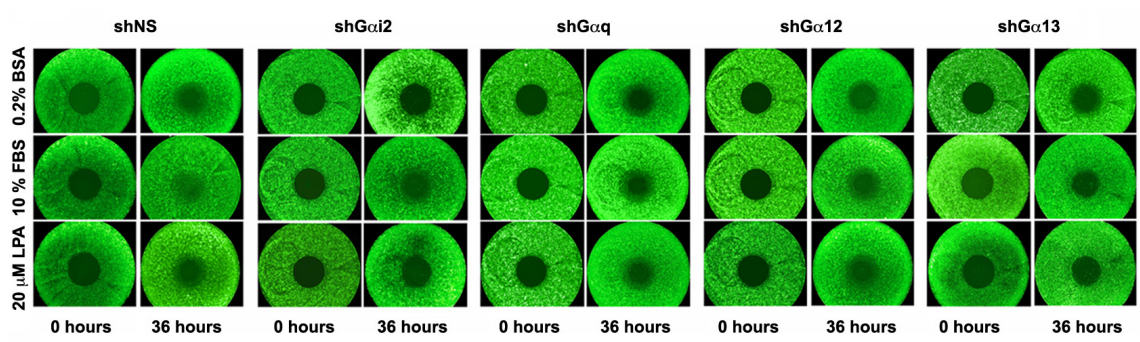

C
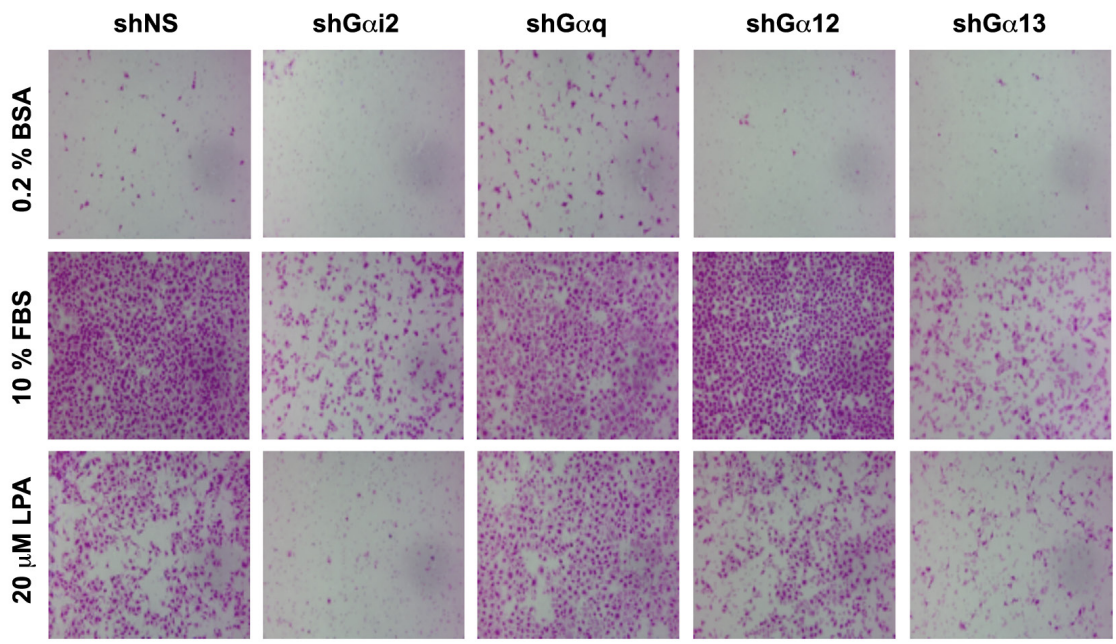

B

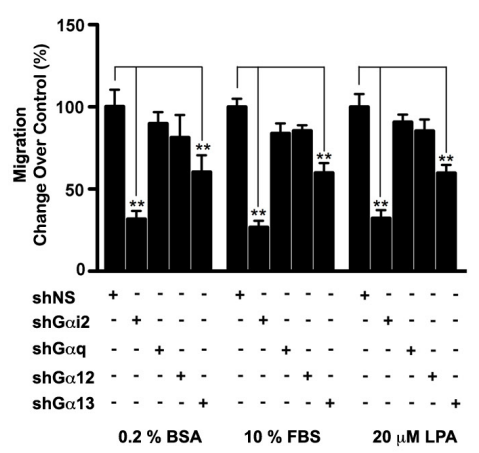

D

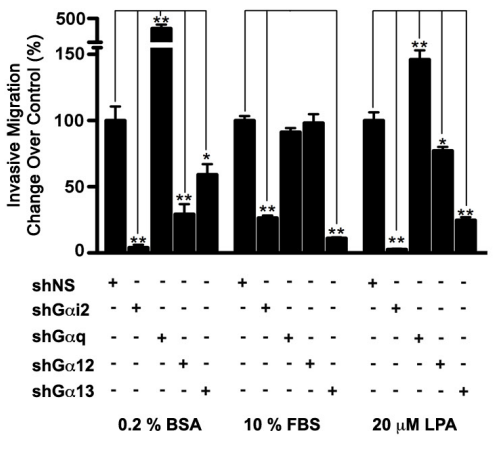

Figure 2: Effect of silencing Ga-subunits on the migration of SKOV3 cells. (A) SKOV3 cells in which Gai2 (shGai2), Gaq (shGaq), Ga12 (shGa12), or Ga13 (shGa13) was silenced along with the cells that express scrambled shRNA (shNS) were plated in 96-well Oris TM cell migration plates $\left(3.5 \times 10^{4}\right.$ cells/well) and labeled with cell tracker Green 5-chloromethylfluorescein diacetate (CMFDA) dye. The cells were serum starved for 18 hours (0 hours) and then stimulated with 10\% FBS or $20 \mu$ M LPA for 36 hours (36 hours). The migration profiles of the transfectants were obtained using the live cell imaging in the Operetta HCS system. The images (2x) presented for 0 hours and 36 hours is a representation of three independent experiments. (B) Migrated cells were quantified in Operetta High Content Imaging System using Harmony image analysis software. Results were presented as the percent change in migration over the shNS control values (Mean \pm SEM, $\mathrm{n}=3$ ). Students-t test was used to obtain the statistical significance and is represented with $* *$ for $\mathrm{p}<0.01$. (C) Invasive potential of the respective Ga-silenced cells were monitored using a Transwell migration assay. Cell culture inserts were coated with rat-tail collagen, type 1 and 4 × $10^{5} \mathrm{t}$ NS control, shG $\alpha 12$, shG $\alpha 13$, shGai2 and shGaq cells were suspended in $200 \mu 1$ serum-free media and placed in the well of the companion plate. The companion plate wells contained $500 \mu \mathrm{L}$ of control serum-free media, or serum-free media complemented with $20 \mu \mathrm{M}$ LPA or $10 \%$ FBS. At the end of 20 hours, the non-migrating cells on the proximal side of the inserts were removed with a cotton swab and the migrated cells on the distal side of the insert were fixed and stained with Hemacolor. Images of migrated cells were obtained from random fields of view at 10X magnification. (D) Migrated cells were enumerated SKOV3 cells expressing scrambled shRNA (shNS) and the results were presented as the percent change in invasive migration over the shNS control values with $10 \%$ FBS. The values are presented mean \pm SEM from three independent experiments. Students-t test was used to obtain the statistical significance and is represented with $*$ for $\mathrm{p}<0.05$ and $* *$ for $\mathrm{p}<0.01$. 
CXCL12-CXCR4 signaling involves both Gai2-mediated mTORC1 signaling [43] and Go12/13 mediated Rho signaling pathways [14] supports this view.

Together with the data on cell proliferation, our results demonstrate that $\mathrm{G} \alpha 12$ and $\mathrm{G} \alpha 13$ are involved in stimulating cell proliferation with no significant role for Gai or Gaq. However, invasive migration of ovarian cancer cells appears to be dependent on Gai2 and Ga13. All of these $\alpha$-subunits have been implicated in the tumorigenesis and tumor progression in many cancers $[29,34,44-46]$. Therefore, we sought to investigate the

A

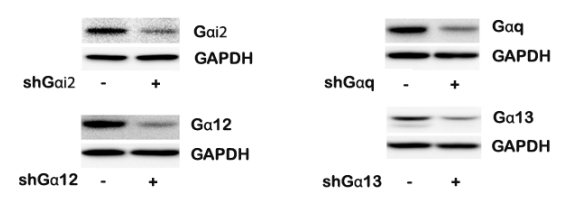

B

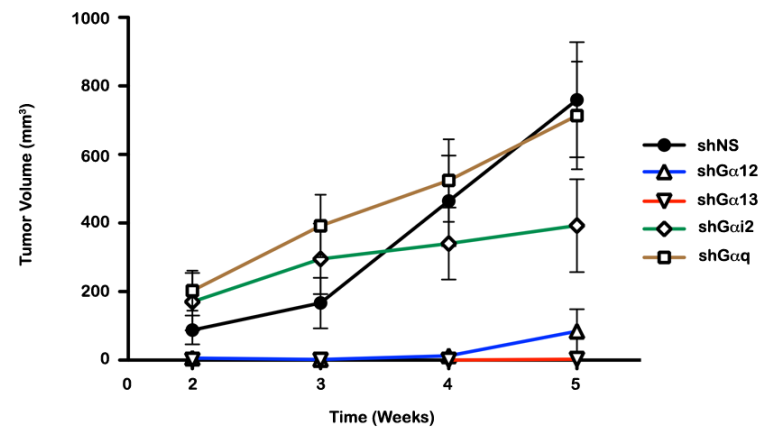

c

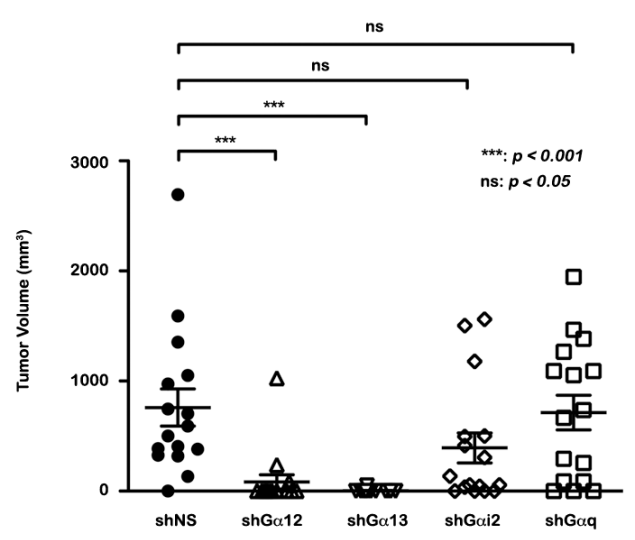

D

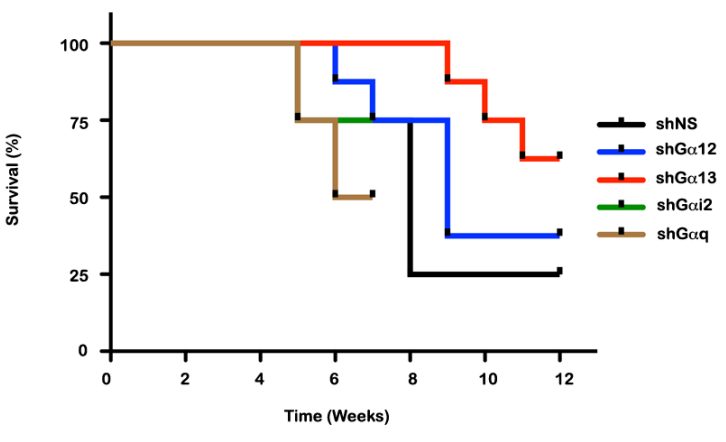

role of these $\mathrm{G} \alpha$-subunits in ovarian cancer growth in vivo by using the respective $\mathrm{G} \alpha$-silenced xenograft tumor mice model. Results from the mice bearing xenograft tumors of the $\mathrm{G} \alpha$-silenced ovarian cancer cells, namely shGai2SKOV3, shGaq-SKOV3, shGa12-SKOV3, and shGa13SKOV3, indicate that the control, Gaq and Gai2 silenced SKOV3 tumors in mice exhibited exponential growth in tumor volume over a period of five weeks (Figure 3B \& $3 \mathrm{C})$. More significantly, a decrease in tumor volume was

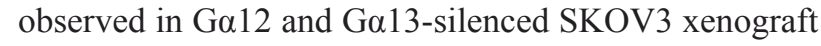
tumors in comparison with the other groups (Figure 3B, C). In this regard, our findings agree to a certain extent with the results obtained with non-small cell lung carcinoma cells, in which the silencing of either Ga12 or Ga13 independently decelerated tumor growth [47]. However, our analysis of animal survival data using Kaplan-Meier plot indicated a more significant role for $\mathrm{G} \alpha 13$ than any other $\alpha$-subunits. As shown in figure 3C, the silencing of Ga13 prolonged the survival of the xenograft tumor bearing mice compared to the ones bearing shGai2, Gaq, or control group. Although, the silencing of $\mathrm{G} \alpha 12$ was not as protective as $\mathrm{G} \alpha 13$, these animals also showed extended survival compared to Gai2, Gaq, or NS-silenced xenograft tumor bearing mice. In fact, the animals bearing Gaqsilenced xenograft tumor showed aggressive tumor growth that led to the euthanization of the animals as early as 7 weeks. Thus, our studies point to a critical role for $\mathrm{G} \alpha 13$ in promoting tumor progression in vivo, especially in ovarian cancer context. Nevertheless, it should be noted here that silencing of $\mathrm{G} \alpha 12$ also led to tumor growth inhibition next only to the silencing of $\mathrm{G} \alpha 13$. Interestingly, the differences in the tumor volumes and tumor growth between shGa12 and shGa13 tumor group animals were quite minimal,

Figure 3: Effect of silencing Ga-subunits on xenograft tumor growth. (A) Mycoplasma free, stably silenced SKOV3 G protein cell lines (shGa12, shGa13, shGai2 and shG $\alpha \mathrm{q}$ ) were ascertained for appropriate $\mathrm{G}$ protein silencing by immunoblotting with $\mathrm{G} \alpha 12, \mathrm{G} \alpha 13, \mathrm{G} \alpha \mathrm{i} 2$ and $\mathrm{G} \alpha \mathrm{q}$ antibodies and GAPDH antibody for monitoring equal loading of lysate protein. (B) $1 \times 10^{6} \mathrm{SKOV} 3 \mathrm{NS}$ control (nonspecific scrambled control) and SKOV3 G protein silenced (shGa12, shGa13, shGai2, shGaq) ovarian cancer cells were subcutaneously injected on the dorsal surface of NU/NU nude mice. The tumor volume of all the

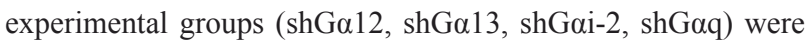
measured over a period of five weeks and assessed in comparison to the NS control group. (C) Mean tumor volumes and individual tumor volumes of each group were assessed between NS control and $\mathrm{G}$ protein silenced groups. Statistical significance for tumor volume differences and mean tumor volumes between the NS control group and the experimental groups were determined using students-t test, $* p<0.05, * * p<0.01$. (D) The percentage of survival of each $\mathrm{G}$ protein silenced group ( $\mathrm{shG} \alpha 12$, shGa13, shGai2, and shGaq) was determined with respect to the control group, by monitoring their survival for a period of 12 weeks. 
which is consistent with the findings that they show $67 \%$ amino acid identity and they are involved in the activation

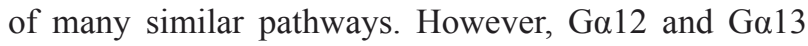
are also involved in the activation of unique pathways of their own. Thus, it is possible that the increased protective effect of Ga13 on tumor bearing animals, compared to Ga12, may be indicative of the unique pathways activated by Ga13. Quantitatively, our results differ by establishing a more dominant role for Ga13. In addition, by assessing the role of other growth promoting $\mathrm{G} \alpha$-subunit in ovarian cancer context in which aberrant signaling by LPARs play a critical role, we firmly establish a determinant role for

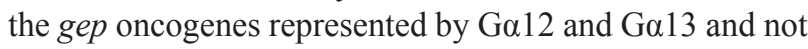
to Gai2. A simple paradigm based on the results presented here along with our previous data would suggest that the increased oncogenicity of G $\alpha 13$ could be associated with its unique ability to stimulate both mitogenic and motogenic pathways. Although mitogenic pathways and motogenic pathways are often mutually exclusive, it is possible that Ga13-mediated effects, on both of these signaling events are temporally regulated. Considering the heterogeneity of tumor cell population in cancer tissue, it is also possible that $\mathrm{G} \alpha 13$ stimulates proliferation or migration in different population of cancer cells, which collectively contribute towards aggressive tumor growth. Further defining of the unique downstream signaling nodes associated with Ga13 signaling axis could unravel novel targets for ovarian cancer therapy and disease management.

\section{MATERIALS \& METHODS}

\section{Cell lines and culture}

Control SKOV3 cell expressing nonspecific scrambled shRNA (SKOV3 NS) and the G $\alpha$-silenced SKOV3 cell lines (shGai2, shGaq, shGa12, and shGa13) were cultured and maintained in Dulbecco's modified Eagle's Medium (DMEM) (Cellgro, Manassas, VA), containing $10 \%$ Fetal Bovine Serum (Gemini BioProducts, West Sacramento, CA), 50 units/mL Penicillin, and $50 \mu \mathrm{g} / \mathrm{mL}$ Streptomycin at $37^{\circ} \mathrm{C}$ in a $5 \% \mathrm{CO} 2$ incubator. Oleoyl (18.1) LPA was obtained from Avanti Polar Lipids (Alabaster, AL) was dissolved in PBS containing $0.1 \% \mathrm{BSA}$ as $10 \mathrm{mM}$ stock solutions, and stored at $-20^{\circ} \mathrm{C}$.

\section{Stable Cell line Generation and Immunoblot analysis}

Non-target control shRNA pLKO.1 vector construct was purchased from Sigma-Aldrich, St. Louis, MO
(SHC002) whereas pLKO.1 vector constructs targeting Gai2 (RHS3979-9596925), Gaq (RHS3979-9604171), Ga12 (RHS3979-98491914), and Ga13 (RHS39799604295) were purchased from Open Biosystems (Lafayette, CO). Stable transfections were performed using Amaxa Biosystems Nucleofector II, according to the instructions of the manufacturer. The stably transfected NS control and G $\alpha$-silenced clones were selected with puromycin (2 $\mu \mathrm{g} / \mathrm{ml}$; MP Biomedicals, Solon, Ohio) and single clones were picked, expanded to obtain stable cell lines. The efficiency of silencing the expression of the respective $\alpha$-subunit was ascertained in the respective stable cell lines using immunoblot analysis, in accordance to our previously published methods[37]. Antibodies to Gai2 (sc-13534), Gaq (sc-393), Ga12 (sc409), Ga13 (sc-410), , were purchased from Santa Cruz Biotechnology Inc, CA, and the GAPDH antibody was purchased from Life Technologies-Ambion (AM4300). Peroxidase-conjugated anti-rabbit IgG (W401B) and antimouse IgG (NA931V) were purchased from Promega Corporation (Madison, WI). The blots were developed using SuperSignal West Pico chemiluminescent substrate (34080) from Perkin Elmer (Waltham, MA) and imaged using Kodak Image Station 4000 MM.

\section{Cell Proliferation Assays}

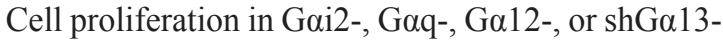
silenced SKOV3 cells along with the control NS SKOV3 cells was monitored by two different assays. A cell count based assay was carried out in which the increase in cell number following the stimulation with $20 \mu \mathrm{M}$ LPA or $10 \%$ FBS. Respective G $\alpha$-silenced SKOV3 cells $\left(5 \times 10^{3}\right.$ cells) along with nonspecific scrambled shRNA expressing control cells were plated in 96-well plates, serum starved for 18 hours, and then stimulated with $10 \%$ FBS or $20 \mu \mathrm{M}$ LPA. Images were obtained at 0, 24 and 48 hours using digital phase contrast imaging in Operetta High Content Imaging System and quantified by Harmony, a high content imaging and analysis Software. To monitor the extent of cell proliferation, the percentage of proliferating cells (S-phase cells) was analyzed by deoxynucleotide (5-ethynyl-2'-deoxyuridine or EdU) incorporation assay, using a Click-iT Plus EdU Alexa Fluor 488 imaging kit (C10637) from Life Technologies (Grand Island, NY). Briefly, $5 \times 10^{3}$ cells of each cell line was plated in 96 well plates, serum starved, stimulated with $10 \%$ FBS or $20 \mu \mathrm{M}$ LPA and incubated with $10 \mu \mathrm{M}$ EdU at 22 hours. After 2 hours of incubation, the EdU incrporated cells were fixed, permeabilized, stained according to the manufacturer's protocols. Proliferating cells versus the total number of DAPI-labeled cells were imaged and quantified in Operetta High Content Imaging System using Harmony, the built-in image analysis software. 


\section{Cell Migration Assay}

Migratory potential was monitored using Oris Cell Migration assay (Platypus Technologies. Madison, WI, \# CMACC5.101). $35 \times 10^{3}$ cells G $\alpha$-silenced SKOV3 cells, along with the control cells were labeled with Cell Tracker Green CMFDA Dye (Life technologies-Molecular Probes, \#C2925) and plated in 96-well Oris TM cell migration plates (CMACC5.101) using manufacturer's protocol. Briefly, the transfectants were serum starved for 18 hours. and the detection zone blocking stoppers were removed. Cells were treated for $1 \mathrm{~h}$ with $0.5 \mu \mathrm{M}$ mitomycin (47589, Calbiochem, La Jolla, CA) to inhibit proliferation and then stimulated with $10 \mu \mathrm{M}$ LPA or $10 \%$ FBS for 36 hours. Pre-migration $(0 \mathrm{hr})$ and migration of cells into the detection zone at $36 \mathrm{hrs}$ were imaged using Operetta High Content Imaging System. Migrated cells were quantified by Harmony image analysis software of the Operetta and the percentile cell migration at $36 \mathrm{hrs}$ over the 0 -hr controls were plotted.

\section{Invasive Migration Assay}

Invasive migration assay was carried in accordance to our previously described $[33,37]$ methods. Cell culture inserts (polyethylene terephthalate membrane with $8.0 \mu \mathrm{m}$ pores \#353097, BD Biosciences, Franklin Lakes, NJ) were coated with rat-tail collagen, type 1 (BD Biosciences) and $4 \times 10^{5}$ cells suspended in $200 \mu \mathrm{L}$ serum-free media were placed in the well of the companion plate. The companion plate wells contained $500 \mu \mathrm{L}$ of control serum-free media and either serum-free media with $10 \mu \mathrm{M}$ LPA or $10 \%$ FBS. At the end of 20 hours, the non-migrating cells on the proximal side of the inserts were removed with a cotton swab and the migrated cells on the distal side of the insert were fixed and stained with Hemacolor (EMD Chemicals, Inc., Gibbstown, NJ). The migrated cells were enumerated with the images obtained from random fields of view at 10X magnification and the results were presented as the percentage of migration.

\section{Animal experiments and ethical compliance}

$\mathrm{Nu} / \mathrm{Nu}$ nude mice (5-6 weeks old) were purchased from Charles River laboratories (Wilmington, MA) and were housed in a barrier facility under 12 hour light/ dark cycle under pathogen free conditions, with food and water ad libitum. All experiments were performed with the approval of the university of Oklahoma Health Science Center institutional animal care and use committee. Mycoplasma free NS-SKOV3 (nonspecific scrambled shRNA control) and shGai2-SKOV3, shGaq-SKOV3, shGa12-SKOV3, or shGa13-SKOV3 cells $\left(1 \mathrm{X} 10^{6}\right)$ were injected subcutaneously on the dorsal surface of NU/NU nude mice to obtain ovarian cancer xenograft tumors. The control and experimental groups were monitored regularly for tumor development, and the tumor volume in all the groups were measured for over a period of five weeks. Animals experiencing pain or cachexic symptoms were euthanized appropriately with the opinion of the institutional veterinarian.

\section{Statistics}

Graph pad prism software (La Jolla, California) was utilized to perform Student's t-test and Kaplan-Meir analysis.

\section{ACKNOWLEDGMENTS}

This work was supported by grants from the National Institutes of Health (CA123233, CA 116984, and GM103639).

\section{CONFLICTS OF INTEREST} interests.

The authors declare that they have no competing

\section{REFERENCES}

1. Siegel RL, Miller KD and Jemal A. Cancer statistics, 2015. CA: a cancer journal for clinicians. 2015; 65(1):5-29.

2. Hatziapostolou M, Polytarchou C, Panutsopulos D, Covic $\mathrm{L}$ and Tsichlis PN. Proteinase-activated receptor-1-triggered activation of tumor progression locus- 2 promotes actin cytoskeleton reorganization and cell migration. Cancer research. 2008; 68(6):1851-1861.

3. Loberg RD, Tantivejkul K, Craig M, Neeley CK and Pienta KJ. PAR1-mediated RhoA activation facilitates CCL2induced chemotaxis in PC-3 cells. Journal of cellular biochemistry. 2007; 101(5):1292-1300.

4. Muppidi JR, Schmitz R, Green JA, Xiao W, Larsen AB, Braun SE, An J, Xu Y, Rosenwald A, Ott G, Gascoyne RD, Rimsza LM, Campo E, Jaffe ES, Delabie J, Smeland EB, et al. Loss of signalling via Galpha13 in germinal centre B-cell-derived lymphoma. Nature. 2014; 516(7530):254258.

5. Patel M, Kawano T, Suzuki N, Hamakubo T, Karginov AV and Kozasa T. Galpha13/PDZ-RhoGEF/RhoA signaling is essential for gastrin-releasing peptide receptor-mediated colon cancer cell migration. Molecular pharmacology. 2014; 86(3):252-262.

6. Gschwind A, Prenzel N and Ullrich A. Lysophosphatidic acid-induced squamous cell carcinoma cell proliferation and motility involves epidermal growth factor receptor signal transactivation. Cancer research. 2002; 62(21):63296336. 
7. Liu Z, Hopkins MM, Zhang Z, Quisenberry CB, Fix LC, Galvan BM and Meier KE. Omega-3 fatty acids and other FFA4 agonists inhibit growth factor signaling in human prostate cancer cells. The Journal of pharmacology and experimental therapeutics. 2015; 352(2):380-394.

8. Stahle M, Veit C, Bachfischer U, Schierling K, Skripczynski B, Hall A, Gierschik P and Giehl K. Mechanisms in LPAinduced tumor cell migration: critical role of phosphorylated ERK. J Cell Sci. 2003; 116(Pt 18):3835-3846.

9. Gomathinayagam R, Muralidharan J, Ha JH, Varadarajalu L and Dhanasekaran DN. Hax-1 is required for Rac1Cortactin interaction and ovarian carcinoma cell migration. Genes \& cancer. 2014; 5(3-4):84-99.

10. Rozengurt E. Early signals in the mitogenic response. Science. 1986; 234(4773):161-166.

11. Gutkind JS. The pathways connecting G protein-coupled receptors to the nucleus through divergent mitogenactivated protein kinase cascades. The Journal of biological chemistry. 1998; 273(4):1839-1842.

12. Attal H, Cohen-Hillel E, Meshel T, Wang JM, Gong W and Ben-Baruch A. Intracellular cross-talk between the GPCR CXCR1 and CXCR2: role of carboxyl terminus phosphorylation sites. Experimental cell research. 2008; 314(2):352-365.

13. Zlotnik A, Burkhardt AM and Homey B. Homeostatic chemokine receptors and organ-specific metastasis. Nature reviews Immunology. 2011; 11(9):597-606.

14. Yagi H, Tan W, Dillenburg-Pilla P, Armando S, Amornphimoltham P, Simaan M, Weigert R, Molinolo AA, Bouvier $M$ and Gutkind JS. A synthetic biology approach reveals a CXCR4-G13-Rho signaling axis driving transendothelial migration of metastatic breast cancer cells. Science signaling. 2011; 4(191):ra60.

15. Richard DE, Vouret-Craviari V and Pouyssegur J. Angiogenesis and G-protein-coupled receptors: signals that bridge the gap. Oncogene. 2001; 20(13):1556-1562.

16. Staller P, Sulitkova J, Lisztwan J, Moch H, Oakeley EJ and Krek W. Chemokine receptor CXCR4 downregulated by von Hippel-Lindau tumour suppressor pVHL. Nature. 2003; 425(6955):307-311.

17. Sosa MS, Lopez-Haber C, Yang C, Wang H, Lemmon MA, Busillo JM, Luo J, Benovic JL, Klein-Szanto A, Yagi H, Gutkind JS, Parsons RE and Kazanietz MG. Identification of the Rac-GEF P-Rex1 as an essential mediator of ErbB signaling in breast cancer. Molecular cell. 2010; 40(6):877892.

18. Goldsmith ZG and Dhanasekaran DN. G protein regulation of MAPK networks. Oncogene. 2007; 26(22):3122-3142.

19. Cao C, Huang X, Han Y, Wan Y, Birnbaumer L, Feng GS, Marshall J, Jiang M and Chu WM. Galpha(i1) and Galpha(i3) are required for epidermal growth factormediated activation of the Akt-mTORC1 pathway. Science signaling. 2009; 2(68):ra17.

20. Garcia-Marcos M, Ghosh P and Farquhar MG. GIV is a nonreceptor GEF for $\mathrm{G}$ alpha $\mathrm{i}$ with a unique motif that regulates Akt signaling. Proceedings of the National Academy of Sciences of the United States of America. 2009; 106(9):3178-3183.

21. Garcia-Marcos M, Ghosh P and Farquhar MG. GIV/Girdin transmits signals from multiple receptors by triggering trimeric $\mathrm{G}$ protein activation. The Journal of biological chemistry. 2015; 290(11):6697-6704.

22. Malbon CC. Beta-catenin, cancer, and G proteins: not just for frizzleds anymore. Sci STKE. 2005; 2005(292):pe35.

23. Turm H, Maoz M, Katz V, Yin YJ, Offermanns S and Bar-Shavit R. Protease-activated receptor-1 (PAR1) acts via a novel Galpha13-dishevelled axis to stabilize betacatenin levels. The Journal of biological chemistry. 2010; 285(20):15137-15148.

24. Kasai K, Takahashi M, Osumi N, Sinnarajah S, Takeo T, Ikeda H, Kehrl JH, Itoh G and Arnheiter H. The G12 family of heterotrimeric $\mathrm{G}$ proteins and Rho GTPase mediate Sonic hedgehog signalling. Genes Cells. 2004; 9(1):49-58.

25. Riobo NA, Saucy B, Dilizio C and Manning DR. Activation of heterotrimeric G proteins by Smoothened. Proceedings of the National Academy of Sciences of the United States of America. 2006; 103(33):12607-12612.

26. Douglas AE, Heim JA, Shen F, Almada LL, Riobo NA, Fernandez-Zapico ME and Manning DR. The alpha subunit of the G protein G13 regulates activity of one or more Gli transcription factors independently of smoothened. The Journal of biological chemistry. 2011; 286(35):3071430722 .

27. Shen F, Cheng L, Douglas AE, Riobo NA and Manning DR. Smoothened is a fully competent activator of the heterotrimeric G protein G(i). Molecular pharmacology. 2013; 83(3):691-697.

28. Regue L, Mou F and Avruch J. G protein-coupled receptors engage the mammalian Hippo pathway through F-actin: F-Actin, assembled in response to Galpha12/13 induced RhoA-GTP, promotes dephosphorylation and activation of the YAP oncogene. Bioessays. 2013; 35(5):430-435.

29. Feng $X$, Chen $Q$ and Gutkind JS. Oncotargeting G proteins: The Hippo in the room. Oncotarget. 2014; 5(22):1099710999.

30. Simoncini T, Scorticati C, Mannella P, Fadiel A, Giretti MS, Fu XD, Baldacci C, Garibaldi S, Caruso A, Fornari L, Naftolin F and Genazzani AR. Estrogen receptor alpha interacts with Galpha13 to drive actin remodeling and endothelial cell migration via the RhoA/Rho kinase/moesin pathway. Mol Endocrinol. 2006; 20(8):1756-1771.

31. Liu J, Youn H, Yang J, Du N, Liu J, Liu H and Li B. G-protein alpha-s and -12 subunits are involved in androgen-stimulated PI3K activation and androgen receptor transactivation in prostate cancer cells. Prostate. 2011; 71(12):1276-1286.

32. Venkatraman $\mathrm{G}$, Benesch $\mathrm{MG}$, Tang $\mathrm{X}$, Dewald $\mathrm{J}$, McMullen TP and Brindley DN. Lysophosphatidate 
signaling stabilizes $\mathrm{Nrf} 2$ and increases the expression of genes involved in drug resistance and oxidative stress responses: implications for cancer treatment. FASEB journal : official publication of the Federation of American Societies for Experimental Biology. 2014.

33. Goldsmith ZG, Ha JH, Jayaraman M and Dhanasekaran DN. Lysophosphatidic Acid Stimulates the Proliferation of Ovarian Cancer Cells via the gep Proto-Oncogene Galpha(12). Genes Cancer. 2011; 2(5):563-575.

34. Gardner JA, Ha JH, Jayaraman $\mathrm{M}$ and Dhanasekaran DN. The gep proto-oncogene Galpha13 mediates lysophosphatidic acid-mediated migration of pancreatic cancer cells. Pancreas. 2013; 42(5):819-828.

35. Mikelis CM, Palmby TR, Simaan M, Li W, Szabo R, Lyons R, Martin D, Yagi H, Fukuhara S, Chikumi H, Galisteo R, Mukouyama YS, Bugge TH and Gutkind JS. PDZ-RhoGEF and LARG are essential for embryonic development and provide a link between thrombin and LPA receptors and Rho activation. The Journal of biological chemistry. 2013; 288(17):12232-12243.

36. Ward JD, Ha JH, Jayaraman $\mathrm{M}$ and Dhanasekaran DN. LPA-mediated migration of ovarian cancer cells involves translocalization of Galphai2 to invadopodia and association with Src and beta-pix. Cancer letters. 2015; 356(2 Pt B):382-391.

37. Radhika V, Onesime D, Ha JH and Dhanasekaran N. Galpha13 stimulates cell migration through cortactininteracting protein Hax-1. J Biol Chem. 2004; 279(47):49406-49413.

38. Dhanasekaran DN. Transducing the signals: a $G$ protein takes a new identity. Sci STKE. 2006; 2006(347):pe31.

39. Hewavitharana $\mathrm{T}$ and Wedegaertner PB. Non-canonical signaling and localizations of heterotrimeric $\mathrm{G}$ proteins. Cellular signalling. 2012; 24(1):25-34.

40. Radhakrishnan R, Ha JH and Dhanasekaran DN. Mitogenic Signaling by the gep Oncogene Involves the Upregulation of S-Phase Kinase-Associated Protein 2. Genes \& cancer.
2010; 1(10):1033-1043.

41. Coffield VM, Helms WS, Jiang Q and Su L. Galpha13 mediates a signal that is essential for proliferation and survival of thymocyte progenitors. The Journal of experimental medicine. 2004; 200(10):1315-1324.

42. Ward JD and Dhanasekaran DN. LPA Stimulates the Phosphorylation of p130Cas via Galphai2 in Ovarian Cancer Cells. Genes \& cancer. 2012; 3(9-10):578-591.

43. Dillenburg-Pilla P, Patel V, Mikelis CM, Zarate-Blades CR, Doci CL, Amornphimoltham P, Wang Z, Martin D, Leelahavanichkul K, Dorsam RT, Masedunskas A, Weigert R, Molinolo AA and Gutkind JS. SDF-1/CXCL12 induces directional cell migration and spontaneous metastasis via a CXCR4/Galphai/mTORC1 axis. FASEB journal : official publication of the Federation of American Societies for Experimental Biology. 2015; 29(3):1056-1068.

44. Sales KU, Friis S, Konkel JE, Godiksen S, Hatakeyama M, Hansen KK, Rogatto SR, Szabo R, Vogel LK, Chen W, Gutkind JS and Bugge TH. Non-hematopoietic PAR-2 is essential for matriptase-driven pre-malignant progression and potentiation of ras-mediated squamous cell carcinogenesis. Oncogene. 2015; 34(3):346-356.

45. Gan CP, Patel V, Mikelis CM, Zain RB, Molinolo AA, Abraham MT, Teo SH, Abdul Rahman ZA, Gutkind JS and Cheong SC. Heterotrimeric G-protein alpha-12 (Galpha12) subunit promotes oral cancer metastasis. Oncotarget. 2014; 5(20):9626-9640.

46. Chia CY, Kumari U and Casey PJ. Breast cancer cell invasion mediated by Galpha12 signaling involves expression of interleukins- 6 and -8 , and matrix metalloproteinase-2. J Mol Signal. 2014; 9:6.

47. Grzelinski M, Pinkenburg O, Buch T, Gold M, Stohr S, Kalwa H, Gudermann T and Aigner A. Critical role of G(alpha)12 and G(alpha)13 for human small cell lung cancer cell proliferation in vitro and tumor growth in vivo. Clinical cancer research : an official journal of the American Association for Cancer Research. 2010; 16(5):1402-1415. 